\title{
Relatively Compact Interactions in Many Particle Systems
}

\author{
J. M. Combes*
}

Received January 6, 1969

\begin{abstract}
For many-particle relatively compact interactions the essential spectrum of the $N$-particle Schrödinger Hamiltonian is shown to consist of a continuum $\left[\Lambda_{N},+\infty\left[\right.\right.$ where $\Lambda_{N}$ is the lowest many-body threshold of the system. This result applies in particular to separable interactions and some spin-orbit couplings. The $N$-particle Green's function is studied with the help of the Weinberg equation for many-body forces whose kernel is shown to be compact in the complementary set of $\left[\Lambda_{N},+\infty[\right.$.
\end{abstract}

\section{Introduction}

It has been shown by HunzIKer [1,] [2] with the help of the Weinberg equation that the spectrum of a Schrödinger Hamiltonian for a system of particles interacting via two-body forces, consists of a continuum lying in $\left[\Lambda_{N},+\infty\right.$ [ where $\Lambda_{N}$ is the lowest many-body threshold of the system and in its complement of eigenvalues with finite multiplicities having $\Lambda_{N}$ as only possible accumulation point. HunzikeR's assumption is that the two-body potentials are locally square-integrable and vanish at infinity. Our aim is to extend these results to general "relatively compact" many-particle interactions. Relative compactness is a very useful concept in the study of Schrödinger Hamiltonians, owing on the one hand to the large number of physically interesting local and velocity dependent interactions with this property, and on the other hand to the fact that from the point of view of perturbation theory it enjoys the same virtues as compactness itself. In particular a modified form of Weyl theorem states the invariance of the essential spectrum under such perturbations [5].

As in [2] we shall use the Weinberg equation; the compactness of its kernel will be established with the help of Theorem 1.1 concerning certain connected products of many-particle operators. We shall briefly discuss the relevance of these equations to solve the bound-state problem.

\section{Kinematies and Mathematical Preliminaries}

We consider a $N$ particle system. We shall denote by $D=\left\{C_{1} ; C_{2} ; C_{k}\right\}$ a decomposition of the system into $k$ disjoint clusters (or fragments) $C_{1}, \ldots, C_{k}$. We shall write $\{N\}$ for the trivial decomposition into one

* Attaché de Recherche au C.N.R.S. 
fragment (also denoted by $\{N\}$ ), $D_{0}$ if each fragment contains only one particle, $\{C\}$ if the only $p$-particle fragment with $p>1$ is $C$. The inclusion $D \subset D^{\prime}$ means that each fragment of the cluster decomposition $D$ is contained in a fragment of $D^{\prime}$.

The total momentum in the laboratory system of the cluster $C$ will be denoted by $P_{C}$, the position of its center of mass by $X_{C}$ and its total mass by $m_{C}$; the set of relative cartesian coordinates of particles in cluster $C$ will be designed by the short-hand notation $X^{\{C\}}$.

The total interaction of the particles in cluster $C$ will be denoted by $V^{\{C\}}$. We shall assume that there is no external field acting on the system; in particular $V^{\{i\}}=0$ for each particle $i$. We define the connected part of the interaction $V^{\{C\}}$ by the recursion formula

$$
V_{\text {conn }}^{\{C\}}=V^{\{C\}}-\sum_{C^{\prime} \subset C} V_{\text {conn }}^{\left\{C^{\prime}\right\}} .
$$

To each cluster decomposition $D$ there corresponds a decomposition of the total kinetic energy $H_{0}^{\prime\{N\}}$ in the form

$$
H_{0}^{\prime\{N\}}=\sum_{C \in D} H_{0}^{\prime\{C\}},
$$

where $H_{0}^{\prime\{C\}}=\sum_{i \in C} \frac{\left|P_{i}\right|^{2}}{2 m_{i}}$ is the kinetic energy for the fragment $C$. It can itself be split into the sum

$$
H_{0}^{\prime\{C\}}=\frac{\left|P_{C}\right|^{2}}{2 m_{C}}+H_{0}^{\{C\}} .
$$

The total energy for cluster $C$ is then

$$
H^{\prime\{C\}}=H_{0}^{\prime\{C\}}+V^{\{C\}}
$$

and in the center of mass system of $C$ it is

$$
H^{\{C\}}=H_{0}^{\{C\}}+V^{\{C\}} .
$$

We shall denote by $\mathscr{H}^{\prime}\{C\}$ the irreducible representation space $L^{2}\left(R^{3 p}\right)$ for the canonical commutation relations of the $p$ particles of cluster $C$. It can be written as

$$
\mathscr{H}^{\prime}\{C\}=\mathscr{H}_{C} \otimes \mathscr{H}^{\{C\}},
$$

where $\mathscr{H}_{C}=L^{2}\left(X_{C}\right)$ and $\mathscr{H}^{\{C\}}=L^{2}\left(X^{\{C\}}\right)$.

Let $C=C_{1} \cup C_{2}$ where $C_{1}$ and $C_{2}$ are disjoint clusters. Then one has

$$
\mathscr{H}^{\{C\}}=\mathscr{H}^{\left\{C_{1}\right\}} \otimes \mathscr{H}^{\left\{C_{2}\right\}} \otimes \mathscr{H}^{\left(C_{1}, C_{2}\right)}
$$

where $\mathscr{H}^{\left(C_{1}, C_{2}\right)}=L^{2}\left(X^{\left(C_{1}, C_{2}\right)}\right)$, with $X^{\left(C_{1}, C_{2}\right)}=X_{C_{1}}-X_{C_{2}}$. This results from (6) if we consider $\mathscr{H}_{C}$ as the representation space a particle with mass $m_{C}$ and rearrange $\mathscr{H}_{C_{1}} \otimes \mathscr{H}_{C_{2}}$ according to the well-known 
reduction to the center of mass system in two-body problems, obtained by introducing relative coordinates. This reduction also leads to the useful formula:

$$
H_{0}^{\{C\}}=H_{0}^{\left\{C_{1}\right\}}+H_{0}^{\left\{C_{2}\right\}}+\frac{\left|P^{\left\langle C_{1}, C_{2}\right)}\right|^{2}}{2 m_{\left(C_{1}, C_{2}\right)}},
$$

where $P^{\left(C_{1}, C_{2}\right)}=\frac{m_{C_{1}} P_{C_{2}}-m_{C_{1}} P_{C_{2}}}{m_{C_{1}}+m_{C_{2}}}$ is the relative momentum of the centers of mass of $C_{1}$ and $C_{2}$, and $m_{\left(C_{1}, C_{2}\right)}$ their reduced mass.

We shall denote by $\mathscr{B}(\mathscr{H})$ (resp. $\mathscr{K}(\mathscr{H})$ ) the algebra of bounded (resp. compact) linear operators on the Hilbert space $\mathscr{H}$ and by $I_{\mathscr{H}}$ its identity operator. Then if $C=C_{1} \cup C_{2}, \mathscr{B}\left(\mathscr{H}^{\left\{C_{1}\right\}}\right)$ has according to (7) a natural isomorphic image in $\left.\mathscr{B}\left(\mathscr{H}^{i}{ }^{i}\right\}\right)$ which is

$$
\mathscr{B}\left(\mathscr{H}\left\{C_{1}\right\}\right) \otimes I_{\mathscr{H}}\left\{C_{2}\right\} \otimes I_{\mathscr{H}}\left(C_{1}, C_{2}\right) .
$$

More generally if we define

$$
\mathscr{H}^{D}=\bigotimes_{C \in D} \mathscr{H}^{\{C\}}
$$

then for $D \subset D^{\prime}$ there is a natural embedding of $\mathscr{B}(\mathscr{H} D)$ into $\mathscr{B}\left(\mathscr{H}^{D^{\prime}}\right)$ obtained step by step from the preceding one. This embedding is norm conserving, and in the following we shall denote by the same symbol the algebra $\mathscr{B}\left(\mathscr{H}^{D}\right.$ ) (as well as its elements) and its image by the above embeddings; when confusion is possible we shall specify the space of definition. It is possible to extend in a unique way these embeddings to unbounded self-adjoint operators for which we shall use the same convention. Furthermore the following relation hold [3] for each cluster decomposition $D$

$$
\mathscr{K}\left(\mathscr{H}^{D}\right)=\bigotimes_{C \in D} \mathscr{K}\left(\mathscr{H}^{\{C\}}\right),
$$

where the right-hand side of $(10)$ denotes the norm closure of the subspace of $\mathscr{K}(\mathscr{H} D)$ consisting of finite linear combinations of operators of the following form: they are the product of all elements of a commuting set of operators obtained by choosing one term in each of the isomorphic images in $\mathscr{K}\left(\mathscr{H}^{D}\right)$ of the algebras $\mathscr{K}\left(\mathscr{H}^{\{C\}}\right)$ involved in (11).

The main theorem needed in the next section is the following:

Theorem 1. Let $D=\left\{C_{1} ; C_{2} ; \ldots ; C_{n}\right\}$ be some cluster decomposition. Let the cluster $C_{0}$ be contained in $\bigcup_{i=1}^{p} C_{i}(p \leqq n)$ and such that $C_{0} \cap C_{i}$ is non empty for all $i=1,2, \ldots$. Let $D^{\prime}=\left\{\bigcup_{i=1}^{p} C_{i} ; C_{p+1} ; \ldots ; C_{n}\right\}$. If $A$ and $B$ are respectively operators in $\mathscr{K}\left(\mathscr{H}^{\{D\}}\right)$ and $\mathscr{K}\left(\mathscr{H}^{\left\{C_{0}\right\}}\right)$, then the product $A B$ belongs to $\mathscr{K}\left(\mathscr{H}^{D^{\prime}}\right)$.

The proof of this theorem is given in [4] for $p=n$. For $p<n$ it is easily deduced from the preceding case owing to identity (10).

20 Commun.math. Phys., Vol. 12 
To conclude this section, we briefly describe the commutant $\mathscr{B}^{\prime}\left(\mathscr{H}^{D}\right)$ of the isomorphic image in $\mathscr{B}\left(\mathscr{H}^{\{N\}}\right)$ of $\mathscr{B}\left(\mathscr{H}^{D}\right)$. Starting from (7) it can be shown that $\mathscr{H}^{\{N\}}$ is the tensor product of $\mathscr{H}^{D}$ and of another Hilbert space which is roughly speaking associated to the relative motion of the centers of mass of clusters belonging to $D$. Then $\mathscr{B}^{\prime}\left(\mathscr{H}^{D}\right)$ is the natural image in $\mathscr{B}\left(\mathscr{H}^{\{N\}}\right)$ of bounded linear operators on this Hilbert space.

\section{Relatively Compact Many-Particle Interactions}

It is shown in [1] that the kernel of the Weinberg equation (see Eq. (20) below] is an operator in the Hilbert-Schmidt class for a system of particle interacting through two-body square-integrable potentials. In [2] a limiting procedure allows HunzIKER to derive the compactness of this kernel if the interactions belong to $L^{2}+L^{\infty}$, and the $L^{\infty}$ component can be chosen arbitrarily small. We describe here a class of perturbations containing the preceding ones which seems to be the maximal class for which the Weinberg kernel is a compact operator in $\mathscr{H}^{\{N\}}$.

Let $H$ be a self-adjoint operator on the Hilbert space $\mathscr{H}$. Its domain $\mathscr{D}(\mathscr{H})$ equipped with the scalar product

$$
(f, g)_{\mathscr{D}(H)}=(H f, H g)_{\mathscr{H}}+(f, g)_{\mathscr{H}}
$$

is itself a Hilbert space since $H$ is closed.

Definition 1. [5]. Let $V$ be some (possibly unbounded) linear operator in $\mathscr{H}$ whose domain contains $\mathscr{D}(H)$. Then $V$ is said to be $H$-compact on $\mathscr{H}$ if its restriction to $\mathscr{D}(H)$ is a compact operator from $\mathscr{D}(H)$ to $\mathscr{H}$.

It is proved in Appendix I that this condition is equivalent to saying that for each $z$ in the resolvent set of $H, V(H-z)^{-1}$ is a compact operator on $\mathscr{H}$. Furthermore either one of these two equivalent conditions implies that for each $\varepsilon>0$ there exists a $b(\varepsilon)>0$ such that for all $f$ in $\mathscr{D}(H)$ one has the inequality

$$
\|V f\| \leqq \varepsilon\|H f\|+b(\varepsilon)\|f\|
$$

that is, $V$ is $H$-bounded with zero relative bound (or as we shall say $H$ - $\varepsilon$ bounded).

We shall require from the interaction operators that they satisfy the

Condition (RC). Each $V_{\text {conn }}^{\{C\}}$ is symmetric and $H_{0}^{\{C\}}$-compact on $\mathscr{H}^{\{C\}}$ where $H_{0}^{\{C\}}$ is the Schrödinger kinetic energy operator.

Obviously compact operators on $\mathscr{H}^{\{C\}}$ are $H_{0}^{\{C\}}$-compact. But there also exists a large class of local and velocity dependent potentials, and among them of differential operators with variable coefficients, having this property; in fact for a $p$-particle cluster, the Hilbert space $\mathscr{D}\left(H_{0}^{\{C\}}\right)$ is identical to the Sobolev space $W_{2}\left(R^{3(p-1)}\right)$ and sufficient conditions 
are known ensuring that the closure of these differential operators are compact operators from this Sobolev space to $L^{2}\left(R^{3(p-1)}\right)$. As an example we mention the following results due to BALSLEV [6]:

Theorem 2. Let $X^{\{C\}}=\left(X_{1}, \ldots, X_{3(p-1)}\right)$ be the $3(p-1)$ dimensional vector describing the relative position of the particles belonging to cluster $C$. Let $P_{k}$ be the differential operator $P_{k}=-i \frac{\partial}{\partial X_{k}}, 1 \leqq k \leqq 3(p-1)$. Suppose that there exists a function $\beta\left(\left|X^{\{C\}}\right|\right)$ such that

iii)

$$
\begin{gathered}
\left|V^{\{C\}}\left(X^{\{C\}}\right)\right|^{2} \leqq \beta\left(\left|X^{\{C\}}\right|\right), \\
\int_{0}^{1} \beta\left(\left|X^{\{C\}}\right|\right) \cdot\left|X^{\{C\}}\right| 3-a d\left|X^{\{C\}}\right|<\infty \quad \text { for some } a>0, \\
\int_{R}^{R+1} \beta\left(\left|X^{\{C\}}\right|\right) d\left|X^{\{C\}}\right| \rightarrow 0 \text { as } \quad R \rightarrow \infty .
\end{gathered}
$$

Then $V_{\text {conn }}^{\{C\}}$ is $H^{\{C\}}$-compact.

If condition (ii) is replaced by

$$
\int_{0}^{1} \beta\left(\left|X^{\{C\}}\right|\right) \cdot\left|X^{\{C\}}\right| 1-a d\left|X^{\{C\}}\right|<\infty \quad \text { for some } a>0
$$

then $V^{\{C\}} \cdot P_{k}$ is $H_{0}^{\{C\}}$-compact for all $k$.

This theorem can be of a particular interest if interactions linear in the momenta, such as spin-orbit couplings, are taken into account. However although the inclusion of spin in this framework presents no major difficulties, a detailed study of these interactions is not the aim of this paper and will be treated in a forthcoming one.

\section{Weinberg Equation for Many-Particle Intractions}

As is shown in Appendix I condition (RC) implies that if $C^{\prime} \supset C$ then $V_{\text {conn }}^{\{C\}}$ is $H_{0}^{\left\{C^{\prime}\right\}}$ - $\varepsilon$-bounded on $\mathscr{H}^{\left\{C^{\prime}\right\}}$. Since perturbation of self-adjoint operators by relatively bounded symmetric operators with relative bound smaller than one does not alter the domain and the self-adjointness [5, Chap. 5, § 4] each $\mathscr{H}^{\{C\}}$ is then a uniquely defined self-adjoint operator with the same domain as $H_{0}^{\{C\}}$. This allows us to write the second resolvent equation

$$
\left(z-H^{\{N\}}\right)^{-1}=\left(z-H_{0}^{\{N\}}\right)^{-1}+\left(z-H_{0}^{\{N\}}\right)^{-1} V^{\{N\}}\left(z-H^{\{N\}}\right)^{-1}
$$

and since $\left\|V_{\text {conn }}^{\{C\}}\left(z-H_{0}^{\{N\}}\right)^{-1}\right\| \leqq \varepsilon+\frac{b(\varepsilon)}{|z|}$ can be made as small as we want for $\operatorname{Re} z$ sufficiently negative, we can find a domain of the complex plane in which the iterations of (14) lead to the norm convergent expansion

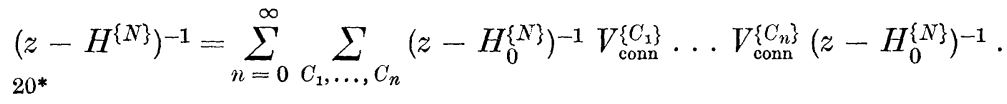


It is then possible to write down the Weinberg equation as is done in [2], with a slight modification due to the presence of many-particle interactions. One draws $N$ horizontal lines representing particles $1,2, \ldots, N$ and one associates to each interaction $V_{\text {conn }}^{\{C\}}$ (here the subscript is essential) a vertical connection of the lines corresponding to particles in $C$. Then we can identify each term in the expansion (15) with a graph obtained by drawing, from the left to the right the vertical connections associated to the interactions of this term, in the order in which they appear in it. In this way the graph

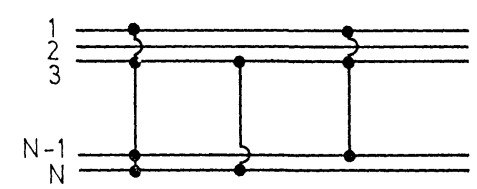

must be identified as

Fig. I

$$
\begin{gathered}
\left(z-H_{0}^{\{N\}}\right)^{-1} V_{\text {conn }}^{(13(N-1) N)}\left(z-H_{0}^{\{N\}}\right)^{-1} \\
V_{\text {conn }}^{(3 N)}\left(z-H_{0}^{\{N\}}\right)^{-1} V_{\text {conn }}^{(13(N-1))}\left(z-H_{6}^{\{N\}}\right)^{-1} .
\end{gathered}
$$

Each graph $G$ defines $k$ maximal disjoint clusters, $k \leqq N$, which consist of particles which can be linked together by some path of the graph formed with horizontal lines or vertical connections. We denote by $D(G)$ the cluster decomposition associated to $G$. We consider now all increasing sequences of cluster decompositions $S=\left(D_{0}, D_{1}, \ldots, D_{k}\right)$ where $D_{i}$ contains $n_{i}$ clusters and is obtained from $D_{i-1}$ by linking $\left[n_{i-1}-n_{i}\right]$ of its clusters; a graph $G$ is said to have connectivity $S$ if $S$ is precisely the sequence of values assumed by the cluster decompositions corresponding to the part of $G$ standing at the left of a vertical line $L$, when $L$ is shifted from the left of $G$ to the right. In fact when $L$ is shifted to the right of some subgraph $G_{i-1}$ of $G$ such that $D\left(G_{i-1}\right)=D_{i-1}$, it can either go over a vertical connection pertaining to some graph $g_{i-1}$ such that $D\left(g_{i-1}\right) \subset D_{i-1}$, or over one linking particles belonging to $\left[n_{i-1}-n_{i}\right]$ different clusters of $D_{i-1}$; in this last case we get a new cluster decomposition $D_{i}$. So the contribution of the graph $G$ to the expansion (15) is the left product of $\left(z-H_{0}^{\{N\}}\right)^{-1}$ by a term of the form

$$
\prod_{i=1}^{k}\left[\begin{array}{l}
\text { any graph } g_{i-1} \\
\text { with } D\left(g_{i-1}\right) \subset D_{i-1}
\end{array}\right] \cdot V_{\text {conn }}^{\left\{C_{i}\right\}}
$$

where $C_{i}$ has a non empty intersection with each of the $\left[n_{i-1}-n_{i}\right]$ old clusters of $D_{i-1}$ and is contained in their union. Let $V_{D_{i}, D_{i-1}}$ denote the sum of the corresponding $V_{\text {conn }}^{\left\{C_{i}\right\}}$ over the clusters $C_{i}$ having this property and define

$$
\begin{aligned}
V^{D} & =\sum_{C \in D} V^{\{C\}}, \\
H^{D} & =H_{0}^{\{N\}}+V^{D}, \\
G_{D}(z) & =\left(z-H^{D}\right)^{-1} .
\end{aligned}
$$


Then summation over all graphs having connectivity $S$ gives the following contribution to (15):

$$
G_{S}(z)=G_{D_{0}}(z) V_{D_{1}, D_{0}} G_{D_{1}}(z) \ldots V_{D_{k, k-1}} G_{D_{k}}(z) \text {. }
$$

It now remains to sum over all sequences $S$. For this we define

$$
\begin{aligned}
& D(z)=\sum_{\left\{S \mid n_{k} \neq 1\right\}} G_{S}(z), \\
& C(z)=\sum_{\left\{S \mid n_{k}=1\right\}} G_{S}(z) .
\end{aligned}
$$

Observing that in $C(z)$ the resolvent $G_{\{N\}}(z)$ can be factored out we obtain the Weinberg equation in the domain of the complex plane in which the iterations of Eq. (14) converge

$$
G_{\{N\}}(z)=D(z)+I(z) G_{\{N\}}(z),
$$

where $I(z)$ is defined on the dense domain $\bigcap_{C} \mathscr{D}\left(V_{\text {conn }}^{\{C\}}\right)$ by

$$
\sum_{\left\{S \mid n_{k}=1\right\}} \prod_{i=1}^{k}\left[G_{D_{i-1}}(z) V_{D_{i}, D_{i-1}}\right] \text {. }
$$

\section{The Discrete Spectrum of $\boldsymbol{H}^{\{N\}}$ Below the Two-Body Threshold}

Let $\Lambda_{N}$ be the greatest lower bound of the spectra of the hamiltonians $H^{D}, D \neq\{N\}$. From our assumption (RC) and the resulting $H_{0}^{\{N\}}-\varepsilon$ boundedness of the interaction $V^{\{N\}}$ it results that $\Lambda_{N}$ is finite. Denote by $\Pi_{A_{N}}$ the complex plane slit along the cut $\left[\Lambda_{N},+\infty\right.$. It is shown in [2] that if the following conditions are satisfied:

1. $I(\bar{z})^{*}=\sum_{\left\{S \mid n_{k}=1\right\}} \prod_{i=1}^{k}\left[V_{D_{i}, D_{i-1}} G_{D_{i-1}}(z)\right]$ and $D(z)$ are analytic families of bounded operators in $\Pi_{\Lambda_{N}}$.

2. For each $z$ in $\Pi_{\Lambda_{N}}, I(\bar{z})^{*}$ is compact. Then the Eq. (20) can be continued analytically to $\Pi_{\Lambda_{N}}$; its solution $G_{\{N\}}(z)$ is given by

$$
G_{\{N\}}(z)=[1-I(z)]^{-1} D(z)
$$

except at possible poles of $[1-I(z)]^{-1}$ which can accumulate only at $\Lambda_{N}$. Here $I(z)=I(z)^{* *}$ is the unique bounded extension of the operator defined in (21).

By the observation that at some bound state energy $E_{0}<\Lambda_{N}$ the spectral projection operator $P\left(E_{0}\right)$ of $\mathscr{H}^{\{N\}}$ satisfies, owing to $(20)$, the relation

$$
P\left(E_{0}\right)=I\left(E_{0}\right) P\left(E_{0}\right)
$$

HUNZIKER concludes that under conditions 1 . and 2. the spectrum of $H^{\{N\}}$ consists of isolated eigenvalues with finite multiplicities which can accumulate only at $\Lambda_{N}$.

Our aim is now to prove 1. and 2. from our condition $(R C)$. This will be derived from the following lemmas 
Lemma 1. Under condition $(R C)$ each product $V_{\text {conn }}^{\{C\}} G_{D}(z)$ is a family of bounded operators on $\mathscr{H}^{\{N\}}$, analytic in the slit complex plane $\Pi_{\Lambda_{N}}$. Hence $D(z)$ and each $I_{S}(\bar{z})^{*}$ also has this property.

Proof. From our condition $(R C)$ and Kato's theorem on relatively bounded perturbations it follows that for each cluster $C$ and decomposition $D$ one has the inclusions

$$
\mathscr{D}\left(H_{0}^{\{N\}}\right)=\mathscr{D}\left(H^{D}\right) \subset \mathscr{D}\left(V_{\text {conn }}^{\{C\}}\right) \subset \mathscr{D}\left(V_{\text {conn }}^{*\{C\}}\right) .
$$

The two norms on $\mathscr{D}\left(H_{0}^{\{N\}}\right)$ determined by the scalar products $(,)_{\mathscr{D}}\left(H_{0}^{\{N\}}\right)$ and $(,)_{\mathscr{D}\left(H^{\{N\}}\right)}$ given by (12) are equivalent, as can be seen from the $H_{0}^{\{N\}}{ }_{-\varepsilon}$-boundedness of the interactions. Hence $G_{D}(z)\left(z \in \Pi_{A_{N}}\right)$ is an analytic family of bounded operators from $\mathscr{H}^{\{N\}}$ to the Hilbert space $\mathscr{D}\left(H_{0}^{\{N\}}\right)$. The $H_{0}^{\{N\}}-\varepsilon$-boundedness of each $V_{\text {conn }}^{\{C\}}$ implies that they are bounded operators from $\mathscr{D}\left(T_{0}^{\{N\}}\right)$ to $\mathscr{H}_{0}^{\{N\}}$. So $V_{\text {conn }}^{\{C\}} G_{D}(z)$ is for $z \in \Pi_{\Lambda_{N}}$ an analytic family of bounded operators from $\mathscr{H}^{\{N\}}$ to $\mathscr{H}^{\{N\}}$. Now $D(z)$ being a sum of products of such operators enjoys the same property. By inclusion (23) the same conclusion holds for

$$
I_{S}(\bar{z})^{*}=V_{\{N\}, D_{N-1}} G_{D_{N-1}}(z) \ldots V_{D_{1}, D_{0}} G_{D_{0}}(z) .
$$

Now the compactness of $I(\bar{z})^{*}$ results from the

Lemma 2. Under condition ( $R C)$, for each $z \in \Pi_{\Lambda_{N}}$ and each sequence $S=\left(D_{0}, D_{1} \ldots,\{N\}\right)$ the operator $I_{S}(\bar{z})^{*}$ is compact.

By the stability of compactness under analytic continuation (see Appendix 2) it will be sufficient to prove compactness of $I_{S}(\bar{z})^{*}$ in the open region $\Delta_{\Lambda_{N}}=\left\{z \mid \operatorname{Re} z<\Lambda_{N}\right\}$. First we recall the integral formula expressing the resolvent of the semi-bounded self-adjoint operator $H$ as a norm convergent integral

$$
(z-H)^{-1}=-\int_{0}^{\infty} e^{z \tau} e^{-H \tau} d t, \quad \operatorname{Re} z<\Lambda,
$$

where $\Lambda$ is the g.1.b. of the spectrum of $H$.

The exponential operator $e^{-H \tau}, \tau>0$, is bounded from $\mathscr{H}$ to $\mathscr{D}(H)$. Hence if $V$ is an $H$ - $\varepsilon$-bounded (resp. $H$-compact) operator in $\mathscr{H}, V e^{-H \tau}$, $t>0$ is a bounded (resp. compact) operator in $\mathscr{H}$. Furthermore $V(H-z)^{-1}$ is the norm limit as $\varepsilon \rightarrow 0$ of $\int_{\varepsilon}^{\infty} e^{z \tau} V e^{-H \tau} d t$. To see this we remark that

$$
\left\|V(H-z)^{-1}-\int_{\varepsilon}^{\infty} e^{+z \tau} V e^{-H \tau} d \tau\right\|=\left\|V\left[e^{-\varepsilon(H-z)}-1\right](H-z)^{-1}\right\| .
$$

The convergence to zero of $\left\|\left(e^{-\varepsilon(H-z)}-1\right)(H-z)^{-1}\right\|$ results from the inequality $\left(1-e^{-\lambda}\right)<\lambda, \lambda>0$. From (13) we deduce that for all $f$ in $\mathscr{H}$ 
and $\eta>0$ there exists $b(\eta)>0$ such that

$$
\begin{aligned}
\left\|V\left[e^{-\varepsilon(H-z)}-1\right](H-z)^{-1} f\right\| & \leqq \frac{\eta}{4}\left\|\left(e^{-(H-z) \varepsilon}-1\right)(H-z)(H-z)^{-1} f\right\| \\
& +(|z|+b(\eta))\left\|\left(e^{-(H-z) \varepsilon}-1\right)(H-z)^{-1} f\right\|
\end{aligned}
$$

Let us choose $\varepsilon_{0}$ such that $\varepsilon<\varepsilon_{0}$ implies $\left\|\left(e^{-(H-z) \varepsilon}-1\right)(H-z)^{-1}\right\|$ $\leqq \frac{\eta}{2}(b(\eta)+|z|)^{-1}$. We can then majorize the left member of $(25)$ by $\eta\|f\|$.

Let us now look at an operator like

$$
V_{\{N\}, D_{k-1}} G_{D_{0}}(z) V_{D_{k-1}, D_{k-2}} \cdots G_{D_{0}}(z) V_{D_{1}, D_{0}} G_{D_{0}}(z)
$$

Since $V_{D_{i}, D_{i-1}}$ is $H_{0}^{\{N\}}-\varepsilon$-bounded one derives from the above considerations that for $\operatorname{Re} z<0,(26)$ can be approximated in the operator norm by the norm convergent integral

$$
\begin{gathered}
\int_{\varepsilon}^{+\infty} \cdots \int_{\varepsilon}^{+\infty}\left(\prod_{i=1}^{k} e^{+z \tau_{i}} d \tau_{i}\right) \cdot V_{\{N\}, D_{k-1}} e^{-H_{0}^{\{N\}} \tau_{k}} V_{D_{k-1, k-2}} \ldots e^{-H_{0}^{\{N\}} \tau_{2}} \\
V_{D_{1}, D_{2}} e^{-H_{0}^{\{N\}} \tau_{1}}
\end{gathered}
$$

A norm convergent integral of compact operators is compact, hence in order to prove compactness of (26) it is sufficient to prove that the integrand is compact on $\mathscr{H}^{\{N\}}$. We recall that $V_{D_{i}, D_{i-1}}$ is a sum of terms $V_{\text {conn }}^{\{C\}}$ over the clusters having a non empty intersection with and only with all the clusters of $D_{i-1}$ which do not belong to $D_{i}$. If $C$ is some of them one has

$$
\begin{aligned}
e^{-H_{0}^{\{N\}} \tau_{i}}= & \left(e^{-\left[\left(C_{n} \sum_{D_{i}} H_{0}^{\{C n\}}\right)-H_{0}^{\{C\}}\right] \tau_{i}}\right)\left(e^{-H_{0}^{\{C\}} \tau_{i}}\right), \\
& \left(e^{-\left[H_{0}^{\{N\}}-{ }_{C_{n} \varepsilon D_{i}} H_{0}^{\{C n\}}\right] \tau_{i}}\right) .
\end{aligned}
$$

Let us look at the left factor on the right-hand side of (28). Let $C_{i}$ be the new cluster of $D_{i}$ (obtained as the union of clusters of $D_{i-1}$ connected by $C$ ) and $C_{i}^{\prime}=C_{i}-C$. Applying (8) to $C_{i}=C \cup C_{i}^{\prime}$ we see that, owing to $(7)$, this first factor contains only operators in $\mathscr{B}\left(\mathscr{H}^{D}\right) \cap \mathscr{B}^{\prime}\left(\mathscr{H}^{\{C\}}\right)$. By the same method the last factor can be shown to be an operator in $\mathscr{B}^{\prime}\left(\mathscr{H}^{D_{i}}\right)$ hence in $\mathscr{B}^{\prime}\left(\mathscr{H}^{D_{i-1}}\right)$. This leads to the following result: Let $A_{i-1}=K_{i-1} B_{i-1}$ where $K_{i-1} \in \mathscr{K}\left(\mathscr{H}^{D_{i-1}}\right)$ and $B_{i-1}$ is some bounded operator in $\mathscr{B}(\mathscr{H}\{N\})$; then $V_{\text {conn }}^{\{C\}} e^{-H_{0}^{\{N\}} \tau_{i}} \cdot A_{i-1}=K_{i} B_{i}$ where $K_{i} \in \mathscr{K}\left(\mathscr{H}^{D^{D}}\right)$ and $\left\|B_{i}\right\| \leqq\left\|B_{i-1}\right\|$. To prove this we use the decom- 
position (28), the compactness in $\mathscr{H}_{0}^{\{C\}}$ of $V_{\text {conn }}^{\{C\}} e^{-H_{0}^{\{C\}} \tau_{i}}$ for any $\tau_{i}>0$, the Theorem 1 and the fact that $\mathscr{K}\left(\mathscr{H}^{D}\right)$ is a two-sided ideal in $\mathscr{B}\left(\mathscr{H}^{D}\right)$. It is now sufficient to use a simple recurrence procedure in order to prove that the integrand is a finite linear combination of operators in $\mathscr{K}\left(\mathscr{H}^{\{N\}}\right)$. Hence (26) is a compact operator.

We now return to $I_{S}^{*}(\bar{z})$. Owing to the inclusion $(23)$ one can omit in its expression the adjunction operation on the potential. Using the second resolvent equation

$$
G_{D}(z)=G_{D_{0}}(z)-G_{D_{0}}(z) V^{D} G_{D}(z)
$$

one can express $I_{S}^{*}(\bar{z})$ as a finite sum of terms having the same form as (26) except that some resolvents $G_{D_{0}}(z)$ in (26) have to be replaced by $G_{D_{0}}(z) V^{D} G_{D}(z)$. But for $\operatorname{Re} z<\Lambda_{N}$, the integral formula (27) still holds for $G_{D}(z), D \neq\{N\}$. And since $V^{D}$ is $H^{D}-\varepsilon$-bounded, these terms can still be approximated in the operator norm by norm convergent integrals of type (27) in which additional factors $V^{D} e^{-H^{D} \tau}$ will have to be interpolated between some terms of (27). But these terms are product of two factors, one in $\mathscr{B}\left(\mathscr{H}^{D}\right)$ and the other in $\mathscr{B}^{\prime}\left(\mathscr{H}^{D}\right)$, so that the same arguments as above will apply in deriving the compactness of the integrand.

\section{The Spectrum of $\boldsymbol{H}^{\{N\}}$}

We now look at the whole spectrum of $H^{\{N\}}$ and show that its essential part consists of the interval $\left[\Lambda_{N},+\infty[\right.$. There exists a $\mathrm{D}=\left\{C_{1}, C_{2}, \ldots, C_{k}\right\}$ such that $A_{N}$ is the g.l.b. of the spectrum of $H_{D}, D \neq\{N\}$. Since this operator has a continuous (and even absolutely continuous) spectrum, WEYL's criterion [8] applies with the result that for each $\lambda$ in $\left[\Lambda_{N},+\infty\right.$ [ there exists an infinite sequence $u_{n}$ in $\mathscr{D}\left(H^{D}\right)$ with $\left\|u_{n}\right\|=1, u_{n}$ tends weakly to zero and $s \cdot \lim \left(H^{D}-\lambda\right) u_{n}=0$. Denote by $f\left(s_{D}\right)$ a vector obtained by applying to $f \in \mathscr{D}\left(H^{\{N\}}\right)$ a translation by $X_{C_{i}}$ of the center of mass of each cluster $C_{i} \in D$ such that $s_{D}=\min _{i \neq j}\left(X_{C_{i}}-X_{C_{j}}\right)$. Then $\left\|\left(H^{D}-\lambda\right) f\left(s_{D}\right)\right\|=\left\|\left(H^{D}-\lambda\right) f\right\|$. We want to show that if

Then :

$$
I_{D}=H^{\{N\}}-H^{D}=\sum_{\substack{C \\ C_{i} \in D}} V_{\text {conn }}^{\{C\}}
$$

$$
\text { s. } \lim _{s_{D} \rightarrow \infty}\left\|I_{D} U_{n}\left(s_{D}\right)\right\|=0 \text {. }
$$

In fact let $V_{\text {conn }}^{\{C\}}$ be one of the interactions in (29); the effect of shifting indefinitely the c.m. of $C_{1}, C_{2}, \ldots, C_{k}$ is to separate more and more the fragments $C_{i}^{\prime}=C \cap C_{i}, i=1,2, \ldots, k$. Owing to the $H_{0}^{\{C\}}$ compactness of $V_{\text {conn }}^{\{C\}}$ in $\mathscr{H}^{\{C\}}$ and to $(7)$ one has then $\lim _{\delta_{D} \rightarrow \infty}\left\|V_{\text {conn }}^{\{C\}}\left(H_{0}^{\{C\}}-z\right)^{-1} f\left(s_{D}\right)\right\|=0$. Since $\mathscr{D}\left(H^{D}\right)=\mathscr{D}\left(H_{0}^{\{C\}}\right)$ one can 
apply this result to $f=\left(H_{0}^{\{C\}}-z\right) u_{n}$ for any $n$ and this implies $\lim _{s_{D} \rightarrow \infty}\left\|V_{\text {conn }}^{\{C\}} u_{n}\left(s_{D}\right)\right\|=0$ and consequently $(30)$. One can then extract a bounded infinite sequence from the set $\left\{u_{n}\left(s_{D}\right)\right\}$ such that the Weyl criterion for $H^{\{N\}}=H^{D}+I^{D}$ can be applied with the result that $\lambda$ belongs to the essential spectrum of $H^{\{N\}}$.

We then summarize all preceding results in the

Theorem 3. Under condition ( $R C$ ) on the interaction, the Hamiltonian $H^{\{N\}}$ is self-adjoint and its domain coincides with $\mathscr{D}\left(H_{0}^{\{N\}}\right)$. Its spectrum consists of a continuum $\left[\Lambda_{N},+\infty\left[\right.\right.$, where $\Lambda_{N}$ is the lowest many-body threshold, and in the real complement of this continuum of eigenvalues of finite multiplicities which can accumulate at most at $\Lambda_{N}$.

Let us mention that for physical interactions $\Lambda_{N}$ is expected to be the two-body threshold but we do not know any method to prove it rigorously.

\section{Concluding Remarks}

The relative compactness condition on interactions is probably not the weakest under which Theorem 3 holds. In particular one would expect that its conclusions remain true for multiplicative interactions in $L_{\text {loc }}^{1}$ tending to zero at infinity and volocity dependent potentials involving the square of momentum operators. The first problems to solve concern then the self-adjointness and the domain of $H^{\{N\}}$ and can probably be overcome as in [6] by the use of quadratic forms instead of operators. It seems on the other hand that $C(z)$ rather than $I(z)$ has some chance to be compact; but it can easily be seen that this is sufficient to derive the results concerning the spectrum of $H^{\{N\}}$.

As was announced in the introduction we briefly discuss the relevance of the Weinberg equation for the bound-state problem. We have seen that every boundstate energy is a pole of $[I-I(z)]^{-1}$. It is well-known that the converse is not true owing to

$$
I-I(z)=D(z)\left(z-H^{\{N\}}\right)
$$

since a zero eigenvalue of $D(z)$ can give a solution of the equation $I(z) f=f$ which would not correspond to a bound state. In fact in the case $N=3$ one has $D(z)=G_{D_{0}}(z)\left[H_{0}^{\{3\}}-\sum_{i<j=1}^{3} t^{(i, j)}(z)-z\right] G_{D_{0}}(z)$, where $t^{(i, j)}(z)$ is the off-shell two-body scattering amplitude. The existence of solutions of the equation $\left[H_{0}^{\{3\}}-\sum_{i<j=1}^{3} t^{(i, j)}(z)-z\right] f=0$ for non real energies has been shown by Federbush [7]. Owing to the analyticity of $I(z)$ such solutions can occur only at some isolated points of the complex plane and it is an open question whether they exist for real negative energies; a proof of the contrary would be a good starting point for an estimate of 
upper-bounds to the binding energy of $N$ particle bound-states with the help of the Weinberg equation.

\section{Appendix 1}

Theorem A.1. Let $H$ be a self-adjoint operator on the Hilbert space $\mathscr{H}$ and $V$ some linear operator whose domain contains $\mathscr{D}(\mathscr{H})$. Then $V$ is $H$-compact on $\mathscr{H}$ if and only if for some $z$ in the resolvent set of $H$ the operator $V(H-z)^{-1}$ is compact; one of these two equivalent conditions implies that $V$ is $H$ - $\varepsilon$ bounded.

Proof. The necessity of the compactness of $V(H-z)^{-1}$ is obvious since $(H-z)^{-1}$ is a bounded operator from $\mathscr{H}$ on $\mathscr{D}(H)$.

Suppose now that $V(H-z)^{-1}$ is compact on $\mathscr{H}$. Then if $f \in \mathscr{D}(H)$ we have $V f=V(H-z)^{-1} h$, with $h=(H-z) f$. If $\left(f_{n}\right)$ is a bounded sequence in $\mathscr{D}(H)$, the corresponding $\left(h_{n}\right)$ is bounded sequence in $\mathscr{H}$. Hence $V f_{n}$ has a limit point in $\mathscr{H}$, which proves $H$-compactness of $V$.

Let $E(\Delta)$ be a projection operator in the spectral family of $H$. For any bounded Borel set $\Delta$ in the spectrum of $H, V E(\Delta)$ is a compact operator on $\mathscr{H}$. Let $\Delta_{n}$ be a sequence of such bounded sets having the property that $E\left(\Delta_{n}\right)$ tends strongly to the identity operator. By a well known property of compact operators $V(H-z)^{-1}\left[1-E\left(\Delta_{n}\right)\right]$ converges in the norm to zero. For $f \in \mathscr{D}(H)$ one has

$$
\|V f\| \leqq\left\|V E\left(\Delta_{n}\right) f\right\|+\left\|V(H-z)^{-1}\left[I-E\left(\Delta_{n}\right)\right](H-z) f\right\| .
$$

For a given $\varepsilon>0$ a suitable choise of an $n_{0}$ gives for $n>n_{0}$

$$
\|V f\| \leqq b_{n}\|f\|+\varepsilon\|(H-z) f\|
$$

which proves $H$ - $\varepsilon$-boundedness of $V$.

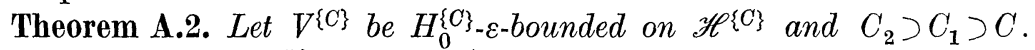
Then $V^{\{C\}}$ acts on $\mathscr{H}^{\left\{C_{2}\right\}}$ as an $H_{0}^{\left\{C_{1}\right\}}{ }_{-\varepsilon}$-bounded operator.

Proof. Let $C_{i}^{\prime}=C_{i}-C$. Then from (7) and (8) we have

$$
\mathscr{H}^{\left\{C_{2}\right\}}=\mathscr{H}^{\{C\}} \otimes \mathscr{H}^{\left\{C_{2}^{\prime}\right\}} \otimes \mathscr{H}^{\left(C, C_{2}^{\prime}\right)} \text { and } H^{\left\{C_{1}\right\}}=H^{\{C\}}+H^{\left\{C_{1}^{\prime}\right\}}+H^{\left(C, C_{1}^{\prime}\right)} \text {. }
$$

We suppose that these self-adjoint operators are acting on $\mathscr{H}^{\left\{C_{2}\right\}}$ and since they are positive we have for all $f$ in the domain of $H_{0}^{\left\{C_{1}\right\}}$

$$
\left\|H_{0}^{\{C\}} f\right\| \leqq\left\|H_{0}^{\left\{C_{1}\right\}} f\right\| .
$$

So that it is sufficient to prove the $H_{0}^{\left\{C_{3}\right.}{ }_{-}$-bounded of $V^{\{C\}}$ on $\mathscr{H}^{\left\{C_{2}\right\}}$. Let $\left(\psi_{m}\right)$ be some orthonormal basis of $\mathscr{H}^{\left\{C_{2}^{\prime}\right\}} \otimes \mathscr{H}^{\left(C, C_{2}^{\prime}\right)}$. Then each $f$ in $\mathscr{H}^{\left\{C_{2}\right\}}$ can be decomposed into the form $f=\sum_{m} \psi_{m} \otimes f_{m}^{\{C\}}$ with $f_{m}^{\{C\}} \in \mathscr{H}^{\{C\}}$, and we have for all $f$ in $\mathscr{D}\left(H_{0}^{\{C\}}\right)$

$$
\left\|H_{0}^{\{C\}} f\right\|^{2}=\sum_{m}\left\|H_{0}^{\{C\}} f_{m}^{\{C\}}\right\|^{2}
$$


so that each $f_{m}^{\{C\}}$ is in the domain of $H_{0}^{\{C\}}$ in $\mathscr{H}^{\{C\}}$ hence in the domain of $V^{\{C\}}$. Now $H_{m}^{\{C\}}$ - $\varepsilon$-boundedness can be written in an equivalent form

$$
\left\|V^{\{C\}} f_{0}^{\{C\}}\right\|^{2} \leqq \varepsilon^{\prime}\left\|H_{0}^{\{C\}} f_{m}^{\{C\}}\right\|^{2}+b^{\prime}\left(\varepsilon^{\prime}\right)\left\|f_{m}^{\{C\}}\right\|^{2}
$$

with $\varepsilon^{\prime}$ as small as we want. Hence $\left\|V^{\{C\}} f\right\|^{2}$ is defined for any $f \in \mathscr{D}\left(H_{0}^{\{C\}}\right)$ in $\mathscr{H}^{\left\{C_{2}\right\}}$ and summation over $m$ gives the desired result.

\section{Appendix 2 (by Hunziker)}

Theorem A.II. Let $A(z)$ be a family of bounded operators on an Hilbert space, analytic in some open connected region $\mathscr{D}$ of the complex plane. If there exists an open subset $\mathcal{O}$ of $\mathscr{D}$ such that $A(z)$ is a compact operator for all $z$ in $\mathcal{O}$, then $A(z)$ is an analytic family of compact operators in $\mathscr{D}$.

Proof. Any point $z_{0}$ in $\mathscr{D}$ can be reached through a chain of open spheres $S_{i}$ contained in $\mathscr{D}$, with centers $z_{i} \in S_{i-1}$ such that $z_{1} \in \mathcal{O}$ and $z_{0} \in S_{n}$. Inside each sphere $S_{i}, A(z)$ is obtained as a norm limit of finite sums $\sum_{k=0}^{K} \frac{\left(z-z_{i}\right)^{k}}{k !} A^{(k)}\left(z_{i}\right)$, where $A^{(k)}\left(z_{i}\right)$ is the generalised derivative of $A\left(z_{i}\right)$

$$
A^{(k)}\left(z_{i}\right)=\frac{k !}{2 i \pi} \int_{C_{i}} \frac{A(z)}{\left(z-z_{i}\right)^{k+1}} d z,
$$

where $C_{i}$ encloses some bounded neibhourhood of $z_{i}$ contained in $\mathscr{D}$. This results easily from usual estimates for the Taylor expansion of analytic functions and Schwartz inequality. Now if the $A(z), z \in S_{i-1}$, are compact operators, these generalised derivatives also are, as can be seen by choosing $C_{i}$ contained in $S_{i-1}$ and using summability of $\|A(z)\|$ on $C_{i}$, which results from analyticity of $A(z)$. Choosing $C_{1}$ contained in the open set $\mathcal{O}$, we derive step by step the compactness of $A\left(z_{0}\right)$.

Acknowledgements. The author is endebted to E. Balslev, A. Grossmann and W. HunzIKer for fruitful discussions.

\section{References}

1. Hunziker, W.: Phys. Rev. 135, B. 800 (1964).

2. - Helv. Phys. Acta 39, 451 (1966).

3. Turumaru, T.: Tôhoku Math. J. 5, 1 (1953).

4. Combes, J. M.: Properties of some connected kernels in multiparticle systems. Preprint.

5. Kato, T.: Perturbation theory for linear operators. Berlin-Heidelberg-New York: Springer 1966.

6. Balslev, E.: Math. Scand. 19, 193 (1966).

7. Federbush, P.: Phys. Rev. 148, 1551 (1966).

8. RiEsz-NAGY: Leçons d'analyse fonctionnelle. Budapest: Akademiai Kiado 1953.

J. M. Combes

Centre de Physique Théorique

C.N.R.S.

31, Chemin J. Aiguier

13 Marseille (9ème), France 